\title{
Test Station for Magnetization Measurements on Large Quantities of Superconducting Strands
}

\author{
S. Le Naour, R. Wolf, J. Billan, and J. Genest
}

\begin{abstract}
In the superconducting main magnets of the Large Hadron Collider (LHC), persistent currents in the superconductor determine the field quality at injection field. For this reason it is necessary to check the magnetization of the cable strands during their production. During four years, this requires measurements of the width of the strand magnetization hysteresis loop at $0.5 \mathrm{~T}, 1.9 \mathrm{~K}$, at a rate of up to eight samples per day. This paper describes the design, construction and the first results of a magnetization test station built for this purpose. The samples are cooled in a cryostat, with a $2-\mathrm{m}$ long elliptic tail. This tail is inserted in a normal conducting dipole magnet with a field between $\pm 1.5 \mathrm{~T}$. Racetrack pick-up coils, integrated in the cryostat, detect the voltage due to flux change, which is then integrated numerically. The sample holder can contain eight strand samples, each $20 \mathrm{~cm}$ long. The test station operates in two modes: either the sample is fixed while the external field is changed, or the sample is moved while the field remains constant. First results of calibration measurements with nickel and niobium are reported.
\end{abstract}

Index Terms-Magnetization, superconductivity, NbTi, LHC.

\section{INTRODUCTION}

\section{A. Why Measure Magnetization}

The proton collider ring LHC [1], which is under construction, uses superconducting magnets operating at a temperature of $1.9 \mathrm{~K}$ to guide the particles. Protons are injected at low field $(0.5 \mathrm{~T})$ in the main bending magnets. At this field, which is $1 / 20$ of the maximum field, persistent current magnetization in the $\mathrm{NbTi}$ filaments of the superconducting magnet cable strands (TABLE I) cause important magnetic field distortions, since the magnetization is high when the field is low. For example, in the LHC dipole at injection the expected [2] sextupole field error due to the magnetization of the filaments is about 10 times as large as the uncertainty due to conductor placement. In addition, variation in the magnetization during production by a manufacturer might induce random field errors in the magnets which decrease the machine performance.

Control limits were therefore introduced into the $\mathrm{LHC}$ TABLE I. CHARACTERISTICS OF LHC MAIN DIPOLE STRANDS.

\begin{tabular}{lccc}
\hline \hline Parameter & $\begin{array}{c}\text { Inner } \\
\text { Strand }\end{array}$ & $\begin{array}{c}\text { Outer } \\
\text { Strand }\end{array}$ & Unit \\
\hline Strand diameter & 1.065 & 0.825 & $\mathrm{~mm}$ \\
Filament diameter & 7 & 6 & $\mu \mathrm{m}$ \\
Number of filaments & 8800 & 6400 & \\
Twist pitch & 18 & 15 & $\mathrm{~mm}$ \\
$\mathrm{Nb} / \mathrm{Ti}$ ratio & $53: 47$ & $53: 47$ & (by weight) \\
Cu:Sc ratio & 1.65 & 1.95 & (by volume) \\
Nb-NbTi ratio & 0.04 & 0.04 & (by volume) \\
\hline \hline
\end{tabular}

Manuscript received September 17, 2000

Authors are with CERN, Geneva, Switzerland.
TABLE II.

TOLERANCES ON STRAND PERSISTENT CURRENT MAGNETIZATION ACCORDING TO THE LHC CABLE SPECIFICATION.

\begin{tabular}{lll}
\hline \hline Parameter & Inner strand & Outer strand \\
\hline M-loop width at $\mathrm{B}=0.5 \mathrm{~T}, \mathrm{~T}=1.9 \mathrm{~K}$ & $<30 \mathrm{mT}$ & $<23 \mathrm{mT}$ \\
Max spread around average value & $\pm 4.5 \%$ & $\pm 4.5 \%$ \\
('Center line') for 1 manufacturer. & & \\
\hline \hline
\end{tabular}

Cable Technical Specification on the width of the magnetization hysteresis loop of the strands (TABLE II). These led to the requirement to construct the magnetization test station described below.

\section{B. Amount of Measurements}

Sampling by one magnetization measurement per billet was thought a reasonable compromise between number and the usefulness of measurements. Although a billet does represent $30-50 \mathrm{~km}$ of strand depending on the manufacturer and strand type, the mechanical and electrical properties over most of a billet is expected to be rather constant. Since about 5500 billets will be manufactured during LHC cable manufacturing, between 5 to 8 magnetization measurements per day at $\mathrm{T}=$ $1.9 \mathrm{~K}$ are then necessary over the 4-year cable production.

\section{MEASUREMENT PRINCIPLE}

To measure the required number of samples it is necessary to measure several samples in one cooldown. The sample length was chosen sufficiently long compared to the twist pitch in order to make measurement of interfilament coupling possible. A simple, straight sample was chosen to minimise mounting time. A normal, accelerator-type dipole magnet provides the high field homogeneity and field volume needed. The samples are cooled to $4.2 \mathrm{~K}$ and $1.9 \mathrm{~K}$ in a cryostat, having a $2 \mathrm{~m}$ long elliptic tail which is inserted in the magnet.

To measure the magnetic moment of the samples, we use four pairs of pick-up coils (Fig. 1, Fig. 2). One pick-up coil consists of two closely spaced racetrack windings. The sample is inserted between these windings. By placing the samples in staggered rows on a movable sample holder it is possible to measure 8 samples with 4 pairs of pick-up coils. The pick-up coils are placed symmetrically in the magnet gap. They are mounted in opposition in order to compensate for the flux variation of the main magnet.

The test station uses two methods (Fig. 1) to measure the magnetization. With the 'static' method the sample remains fixed while the external field is changed, and the voltage due to the flux change is measured with the help of sets of pick-up coils. The magnetization is determined by numeric integration 


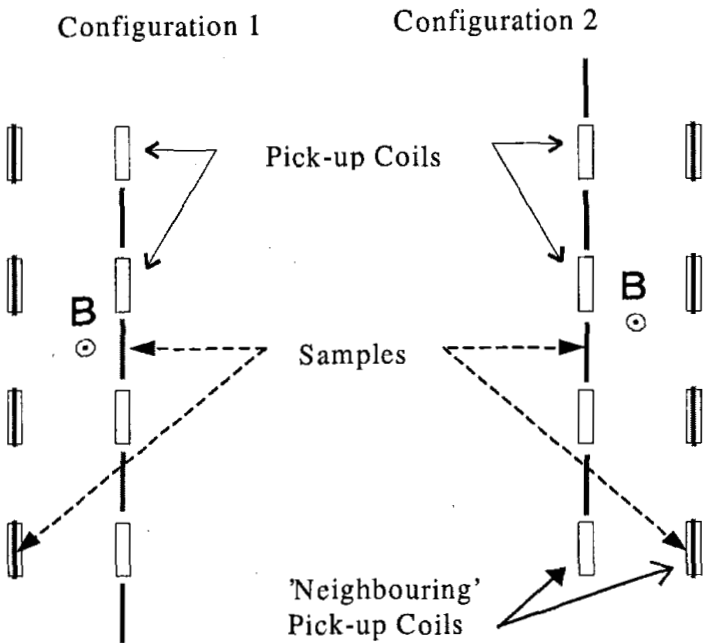

Fig. 1. The samples move in and out of the fixed 'racetrack' pick-up coils. In configuration 1 four samples are inside the pick-up coils. In configuration 2 the other four samples are inside the neighbouring pick-up coils. The magnetic field $B$ is perpendicular to the samples.

of the voltage after the measurement. With the 'kinetic' method the previously magnetized sample is moved through the pick-up coils, while the field remains constant. An actuator on the top of the cryostat moves the sample.

\section{A. Static Method}

The advantage of this method in respect to the kinetic one is that it can be used for determining the magnetization due to currents between the filaments of the strands. Four samples, each inside a respective pick-up coil are, measured simultaneously with the magnetic field performing a prescribed cycle (Configuration 1, Fig. 1). The flux change due to the changing field of the magnet is eliminated (Fig. 2) by subtracting the signal of a neighbouring empty pick-up coil. The procedure is then repeated by changing the position of the samples so that the remaining four are now inside the previously empty coils (Configuration 2, Fig. 1).

\section{B. Kinetic Method.}

For this method the signal level is much higher than for the

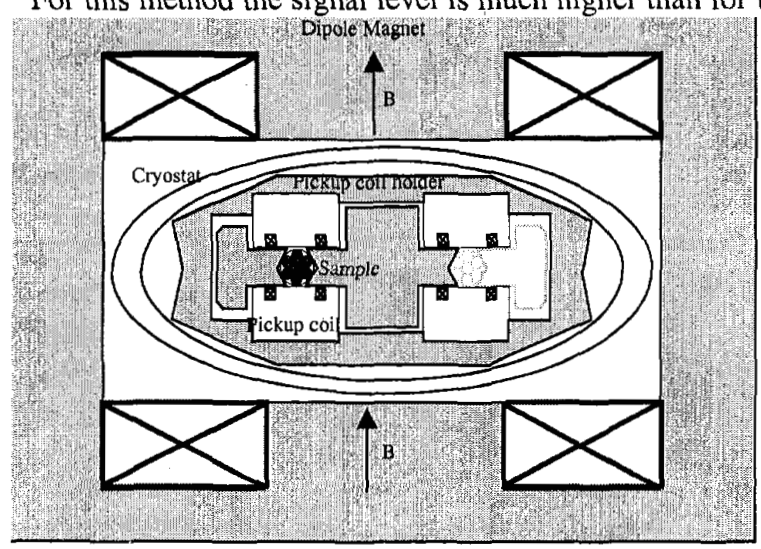

Fig. 2. Sketch of the position of a pair of pick-up coils inside the dipole magnet. The sample is inserted in one pick-up coil. The neighbouring empty pick-up coil is used as a compensation coil. The sample is fixed on a sample holder which can move inside the pick-up coil holder. static method. It is therefore less sensitive to noise and thermal voltages. The sample is displaced in and out of a pick-up coil, changing from configuration 1 to 2 and then back to 1 again (Fig. 1) in about two seconds. The method is too slow to measure complete magnetization hysteresis curves but it meets the simple requirements of the LHC Cable specification (TABLE II).

\section{CONSTRUCTIONAL DETAILS}

\section{A. The Cryostat}

The cryostat (Fig. 3) consists of a $2 \mathrm{~m}$ long 316LN stainless steel elliptical tail and a much wider upper body. The upper body contains up to 50 litres of helium, necessary for having sufficient measurement time at $1.9 \mathrm{~K}$. The elliptical tail dimensions are $48 \mathrm{~mm} \times 120 \mathrm{~mm}$. It has a silverplated vacuum space of $5 \mathrm{~mm}$ and is insulated by two layers of superinsulation. The heat leak at 4.2 and $1.9 \mathrm{~K}$ is $5 \mathrm{~W}$ for the whole set-up and $2 \mathrm{~W} / \mathrm{m}$ in the tail. This permits a measurement time of 4 hours at $1.9 \mathrm{~K}$. Cooldown from 4.2 to $1.9 \mathrm{~K}$ is done by pumping on the helium bath. The cooldown time from 4.2 to $1.9 \mathrm{~K}$ is about an hour.

\section{B. The Magnet and its Power Converter}

The magnet (TABLE III) is a normal, H-type accelerator magnet with an iron, laminated iron yoke. The power converter can deliver $\pm 375 \mathrm{~A}$ at $70 \mathrm{~V}$. This makes it possible to measure magnetization in a field range of $\pm 1.5 \mathrm{~T}$. The homogeneity of the field is sufficient to allow movements of $\pm 1 \mathrm{~mm}$ of the measuring coil assembly without disturbing the reproducibility of the measurements.

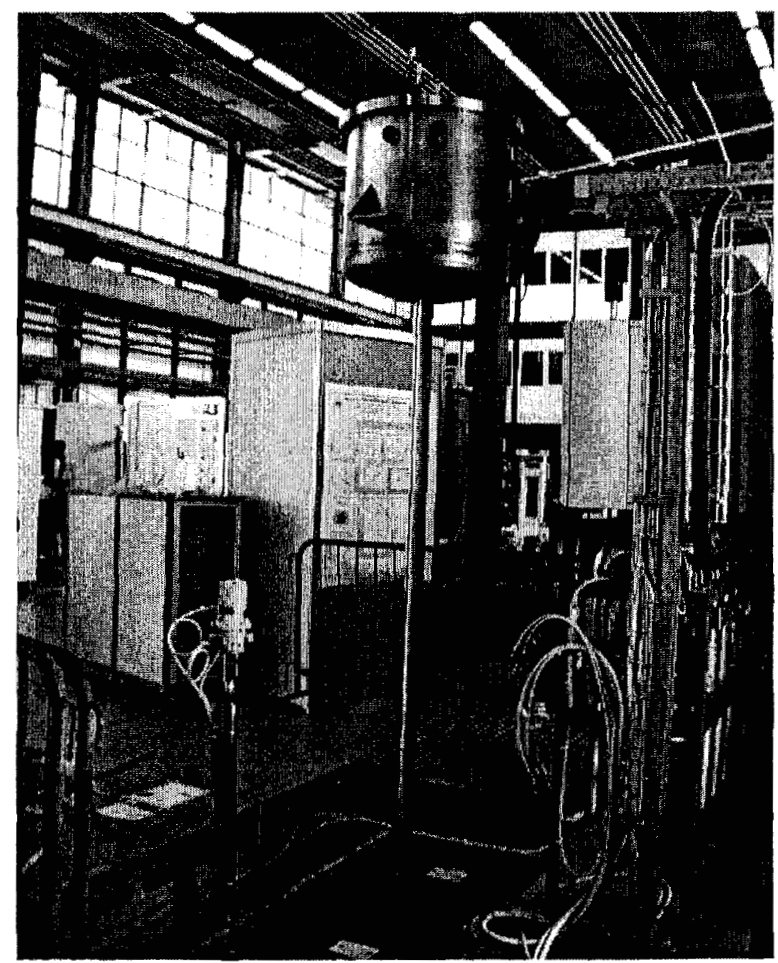

Fig. 3. The Cryostat during mounting inside the dipole magnet. The magnet is inside the trench in the floor. 
TABLE III

MAGNET PARAMETERS

\begin{tabular}{|c|c|c|}
\hline Parameter & Value & Units \\
\hline gap height & 50 & $\mathrm{~mm}$ \\
\hline gap width & 200 & $\mathrm{~mm}$ \\
\hline length & 1.91 & $\mathrm{~m}$ \\
\hline Field at $330 \mathrm{~A}$ & 1.45 & $\mathrm{~T}$ \\
\hline $\begin{array}{l}\text { Field inhomogeneity at } B=0.5 \mathrm{~T} \text { on the } \\
\text { magnet mid plane at } x=13 \mathrm{~mm}\end{array}$ & $0.3 * 10^{-4}$ & \\
\hline Field inhomogeneity over the length & $10^{-3}$ & \\
\hline Remanent field & 1.6 & $\mathrm{mT}$ \\
\hline
\end{tabular}

\section{Measuring coil assembly}

The two coils which form a pick-up coil are spaced by $4 \mathrm{~mm}$. They need to be placed as close as possible to the sample in order to pick up as much magnetic flux as possible (Fig. 4). These coils, above and below the sample are not only needed so that the sample can move in and out of the pick-up coil, but also to limit signal change due to small movements of the coils or samples. The neighbouring pick-up coil is placed at a distance of $26 \mathrm{~mm}$ (Fig. 1, Fig. 2). TABLE IV shows sensitivity to displacement errors and to changes in permeability of the yoke.

The coils consist of 1000 turns of a $32 \mu \mathrm{m}$ diameter copper wire wound in a $1 \mathrm{~mm}$ groove in a fibreglass-epoxy core, as shown in Fig. 5. They are $180 \mathrm{~mm}$ long. Forty coils have been wound, of which 16 have been selected after a coil surface measurement and grouped in pairs of less than $10^{-3}$ difference. The groove wall thickness near the sample was machined to $0.3 \mathrm{~mm}$ thickness in order to decrease the distance to the sample as much as possible.

The connection in series and in opposition of the pick-up coils is made at the top of the measuring coil assembly. In this way the soldered contacts remain in the liquid helium bath and thermal voltages remain low.

\section{The Sample Holder and its Actuator.}

Normally the coil assembly remains in the cryostat. The sample holder slides inside the coil assembly on PEEK inserts. The sample holder consists of a long, fiberglass body

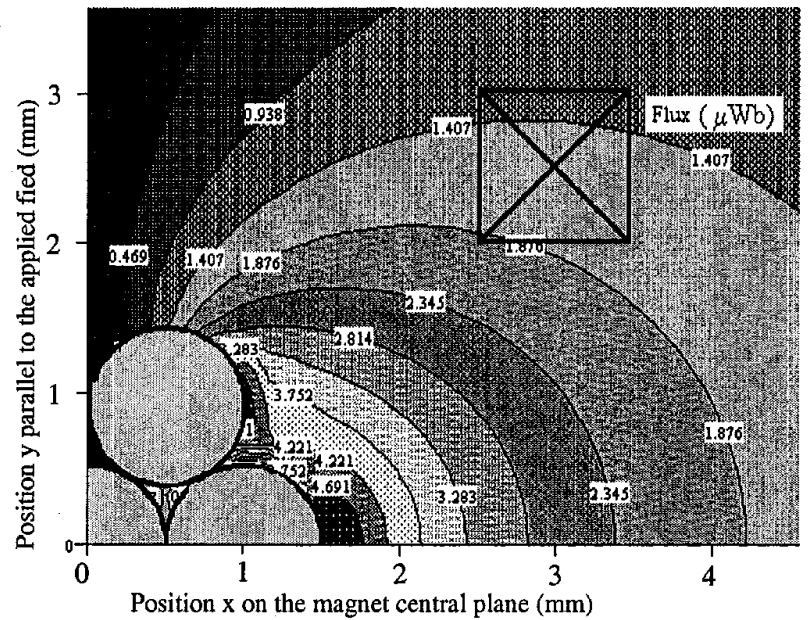

Fig. 4. Field lines due to a magnetized 7-strand sample $\left(\mu_{0} \mathrm{M}=20 \mathrm{mT}\right)$ Only the upper right quadrant is shown. The numbers on the field lines give the flux through one turn of a pickup coil mounted parallel to the magnet plane. The coil winding is mounted as close as possible to the sample (square).
TABLE IV

MEASUREMENT ERROR FOR COIL DISPLACEMENT AND YOKE PERMEABLITYY

\begin{tabular}{lc}
\hline \hline Perturbation & $\begin{array}{c}\text { Change in measured magnetization } \\
\text { relative to the initial value }\end{array}$ \\
\hline coil movement $0.5 \mathrm{~mm}^{(*)}$ & $-1 * 10^{-2}$ \\
coil rotated by $50 \mathrm{mrad}^{(*)}$ & $-1.2 * 10^{-3}$ \\
Change in permeability from & $-3 * 10^{-3}$ \\
$\infty$ to 10 & \\
$\left(^{*}\right)$ These changes are proportional to the square of the perturbation. \\
\hline \hline
\end{tabular}

with eight clamps to hold the samples. The material is the same as that of the coil assembly. The sample holder can be moved over distance of $20 \mathrm{~cm}$ by a stepping motor mounted on the top of the cryostat. The torque of this motor $(4 \mathrm{Nm})$, combined with a torque reducer of $5: 1$ is sufficient to overcome friction and the pressure difference of almost 1 atmosphere where measuring at $\mathrm{T}=1.9 \mathrm{~K}$. Leak tightness is assured by a bellow on the top plate of the cryostat.

\section{E. Sample Mounting}

The sample consists of a bundle of seven $20 \mathrm{~cm}$ long strands (Fig. 2). A special jig was constructed which clamps the seven strands together and wraps adhesive tape around them. The sample is then cut to the right length with a diamond wire saw, which gives a very clean cut on the ends of the sample. We estimate that it will take 40 minutes to mount all the eight samples.

\section{F. Instrumentation and Software}

For the static method the induced voltage is measured with a @Keithley model 2182 nanovoltmeter. This voltmeter is suitable for low voltage measurements with a high accuracy. For an integration time of $60 \mathrm{~ms}$, the noise is $40-50 \mathrm{nV}$ peak to peak. Four nanovoltmeters are used to measure the flux change of the 4 samples inserted in the pick-up coils.

For the kinetic method the voltage is measured with a CKeithley model 2001 multimeter completed with a 10 channel scanner card. The first channel is connected to the DCCT of the power supply of the main magnet and the other
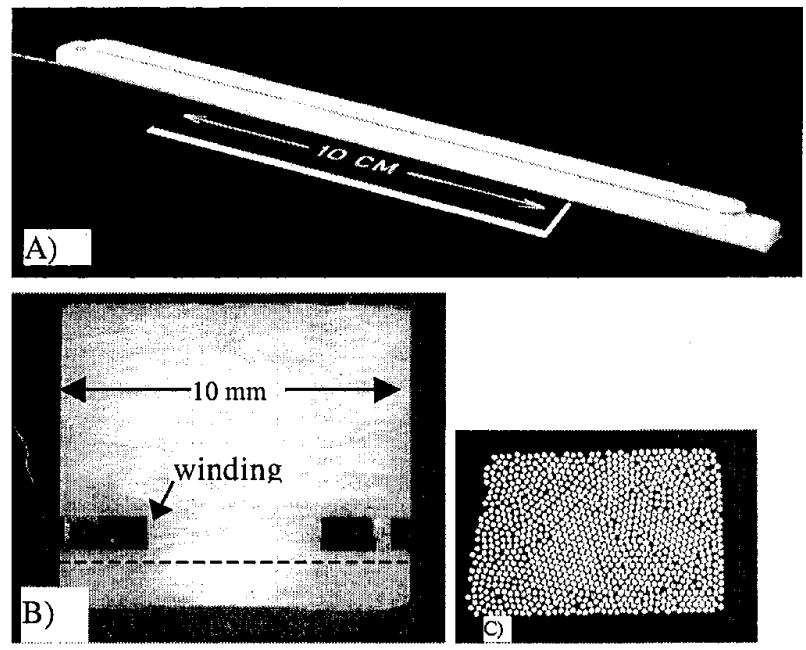

Fig. 5. A) One winding of a pick-up coil. B) A cut showing the crosssection of the core, before maching to the dotted line. C) Cross-section of winding. 
channels are used for the 8 pick-up coils. This multimeter also gives the time when the measurement is done.

Analog integrators are not used The magnetization is obtained by numeric integration. That is why, during the measurements the voltmeters are trigged with the best compromise between the sampling rate and the integration time for a voltage sample.

In the static mode, the 4 nanovoltmeters and the multimeter reading the DCCT are trigged simultaneously via a master trigger with a reading rate of 3.3 readings/s for an integrating time of $60 \mathrm{~ms} /$ reading.

In the kinetic mode, the multimeter uses internal triggering and performs 103 readings $/ \mathrm{s}$ with an integrating time of $2 \mathrm{~ms}$. To obtain these reading frequencies, the data is stored into the internal buffer of each apparatus during the measurements.

An additional multimeter (CKeithley model 2000-20 completed with a 20 channel scanner card measures regularly the voltage relating to temperatures, pressure, liquid helium level, hall probes, current in probes.

All instruments (multimeters, nanovoltmeters, power supplies and actuator) are connected via a GPIB bus and are driven with a National Instrument LabVIEW ${ }^{\mathrm{TM}}$ program. The program allows the synchronisation of all apparatus for the measurements and performs the data acquisition.

\section{MEASUREMENTS}

\section{A. Calibration for the Kinetic Method}

In the saturated state, the nickel magnetization $\mu_{0} M_{\mathrm{s}}$ is $641 \mathrm{mT}$ at $0 \mathrm{~K}[4]$. A first calibration was made with nickel samples with the kinetic method. Nickel rods, $3.5 \mathrm{~mm}$ in diameter, are placed in staggered row on the sample holder and the station is cooled down at $4.2 \mathrm{~K}$.

The magnetic field is fixed at $1 \mathrm{~T}$ and the samples are moved in and out of the pick-up coils with a speed of $200 \mathrm{~mm} / \mathrm{s}$. During the motion the average value of the voltage measured is $167.2 \mathrm{mV}$. The flux change $\Delta \phi_{s}$, calculated by the integration of the signal with a simple trapezium method, is $127.2 \mathrm{mV}$ s for a single motion up or down (Fig. 6). A good reproducibility of this result is obtained, with a maximum difference of $0.7 \%$.

\section{B. Calibration for the Static Method}

Below the first critical magnetic field, superconductors are

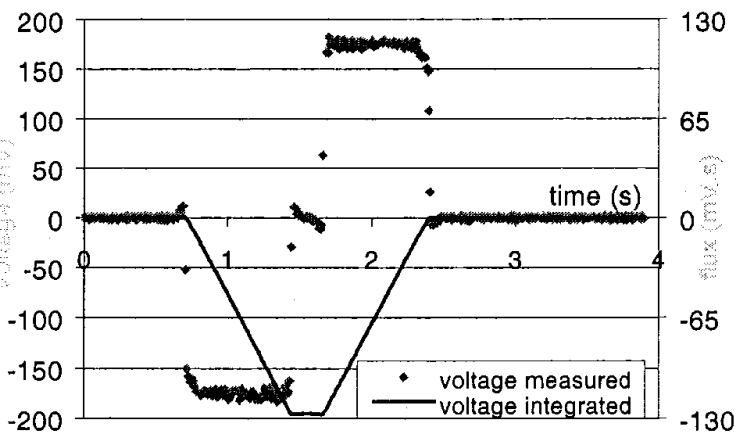

Fig. 6: Signal and integrated signal of a nickel rod in kinetic mode.

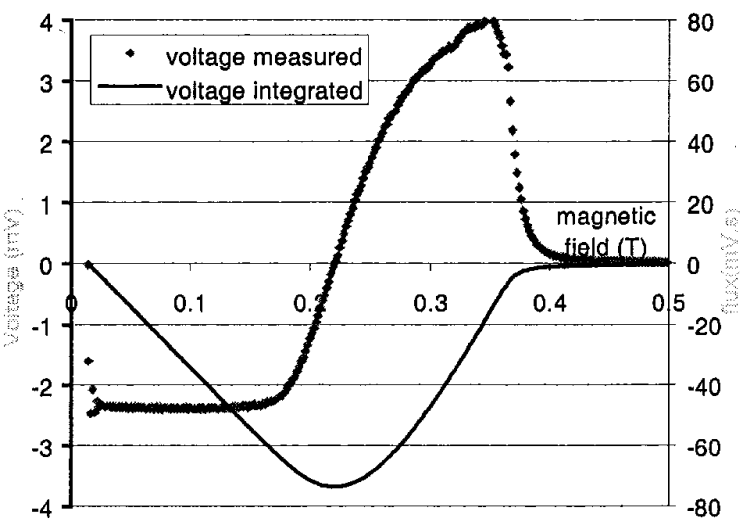

Fig. 7 Signal and integrated signal of niobium using the static method

perfectly diamagnetic. Due to the demagnetization field, the magnetization $\mu_{0} M$ of a long niobium rod is just $-2 \mathrm{~B}_{\mathrm{a}}$, where $\mathrm{B}_{\mathrm{a}}$ is the applied field.

For a magnetic field change rate of $5.933 \mathrm{mT} / \mathrm{s}$, the voltage measured is $-2.37 \mathrm{mV}$ at low field (Fig. 7). The slope $\mathrm{d} \phi / \mathrm{dB}$ of the integrated signal is $-0.400 \mathrm{~V} . \mathrm{s} / \mathrm{T}$.

When we compare the two methods we find that for the kinetic method the calibration factor $\mu_{0} M_{\mathrm{s}} / \Delta \phi_{\mathrm{s}}$ is $5.04 \mathrm{~m}^{-2}$ $(641 \mathrm{mT} / 127.2 \mathrm{mV} \mathrm{s})$ and for the static method the calibration factor $-2 /(\mathrm{d} \phi / \mathrm{dB})$ is $5.00 \mathrm{~m}^{-2}\left(-2 /-0.400 \mathrm{~m}^{2}\right)$.In fact, we expect the calibration factor for the kinetic mode to be somewhat higher, due to the slightly different magnetic coupling to neighbouring samples.

The value for the nickel saturation magnetization however needs confirmation.

\section{CONCLUSIONS}

A magnetization test station for acceptance testing of LHC strands is now being commissioned. The first calibration measurements on nickel and niobium indicate that the test station should work as expected. The innovative feature of this test station is the flexibility provided by the combination of a moving sample magnetometer and measurement in changing field with a fixed sample. The samples are easy to mount.

\section{ACKNOWLEDGEMENT}

The authors thank Mrs. L. Gaborit and R. Beltron for making the pick-up coils, Y. Cuvet for design work, Th. Porrin for software and $\mathrm{Ph}$. Suaton for preparing the experiment.

\section{REFERENCES}

[1] "The Large Hadron Collider, Conceptual Design", CERN/AC/95-05, 20 October 1995.

[2] S. Le Naour, R. Wolf, CERN, 1 Aug. 2000 "The Expected Persistent Current Field Errors in the LHC Main Dipole and Quadrupole", $L H C$ Project-Note-230, CERN, 1 Aug. 2000

[3] S. Le Naour, L. Oberli, R. Wolf, R. Puzniak, R. Szewezyk, A Wisniewski, A. Fikis, H. Foitl, M. Kirchmayr, "Magnetization measurements on LHC superconducting strands", IEEE Trans. Appl. Superconduct. 9 2, pt.2 1999 1763-1766.

[4] Handbook of Chemistry and Physics, p12-119, 80th edition, CRC press, 1999 